\title{
Anti-Fatigue Properties of Cultivated Wild Ginseng Distilled Extract and Its Active Component Panaxydol in Rats
}

\author{
Il-Soo Shin ${ }^{1+}$, Do-Hee Kim ${ }^{2+}$, Eun Young Jang ${ }^{2,3+}$, Hee Young Kim ${ }^{2 *}$, Hwa-Seung \\ $\mathrm{Yoo}^{1 *}$
}

${ }^{1}$ College of Korean Medicine, Daejeon University, Daejeon 34520, South Korea

${ }^{2}$ College of Korean Medicine, Daegu Haany University, Daegu 42158, South Korea

${ }^{3}$ Research Center for Convergence Toxicology, Korea Institute of Toxicology, 141 Gajeong-ro, Yuseong-gu, Daejeon 34114, South Korea

\section{Key Words}

ginseng, fatigue, force swimming test, lactate dehydro-

genase, panaxydol

\section{Abbreviations \\ BUN blood urea nitrogen \\ cWG cultivated wild ginseng \\ FST forced swimming test \\ i.p. intraperitoneally \\ LAC lactate acid \\ LDH lactate dehydrogenase \\ LPS lipopolysaccharide}

\begin{abstract}
Objectives: Cultivated wild ginseng (cWG), called SanYangSanSam, has been used clinically in patients with chronic fatigue in Korea. Little is known about effects of the ginseng distilled (volatile) components produced during evaporizaiton. Recently, we first identified one major component from cWG distilled extract, panaxydol, by using mass spectrometry. However, functional
\end{abstract}

Received: Apr 16, 2019 Reviewed: Apr 22, 2019 Accepted: May 20, 2019

$(0$ This is an Open-Access article distributed under the terms of the Creative Common Attribution Non-Commercial License (http://creativecommons.org/licenses/by-nc/4.0/) which permits unrestricted noncommercial use, distribution, and reproduction in any medium, provided the original work is properly cited.

( This paper meets the requirements of KS X ISO 9706, ISO 9706-1994 and ANSI/NISO Z39.48-1992 (Permanence of Paper). properties of cWG distilled extract and panaxydol remains elusive. Therefore, the present study evaluated the effect of cWG distilled extract or panaxydol on exercise-induced fatigue in rats.

Methods: Fatigue was induced by forced swimming and the immobility time was analyzed in male Sprague-Dawley rats. The animals received intraperitoneally either vehicle, cWG distilled extract, or panaxydol $10 \mathrm{~min}$ prior to beginning of the forced swimming test (FST) once daily for 5 days. After the FST on day 5 , we also analyzed fatigue-related biochemical levels including blood urea nitrogen (BUN), lactate acid (LAC), and lactate dehydrogenase (LDH) in serum and levels of glycogen in liver and soleus muscle.

Results: The forced swimming time in cWG distilled extract $(0.6 \mathrm{~mL} / \mathrm{kg})$-treated group was significantly longer than that of control group on day 4 and 5. Panaxydol ( 0.1 and $0.25 \mathrm{mg} / \mathrm{kg}$ )-treated groups showed significantly enhanced performance in the forced swimming, compared to control. In addition, a significant decrease in serum LDH level was found in panaxydol-treated group, while there were no alternations in levels of serum BUN and LAC and glycogen in liver or soleus muscle.

Conclusion: The present study demonstrated cWG

\section{"Corresponding Author}

Hwa-Seung Yoo. East-West Cancer Center, Daejeon University, Daejeon 34520 , South Korea.

Tel: +82-42-470-9132 Fax:+82-42-470-9006 E-mal: altyhs@dju.kr

Hee Young Kim. Ph.D. Department of Physiology, College of Korean Medicine, Daegu Haany University, Daegu 42158, South Korea.

Tel: +82-53-770-2256 Fax: +82-53-768-6340 E-mail: hykim@dhu.ac.kr

$\dagger$ These authors equally contributed to this work. 
distilled extract and its active component panaxydol have a function of anti-fatigue.

\section{Introduction}

Fatigue is a feeling of extreme physical or mental tiredness and exhaustion as one of the common physiological reactions resulting from severe stress, hard physical or mental work. Fatigue may be associated with many disorders and mainly caused by the depletion of energy sources which include the accumulation of the end products of fatigue, the decrease in liver glycogen consumption [1]. In particular, physical fatigue is the transient inability of a muscle to maintain optimal physical performance and induced by intense physical exercise frequently leads to a deterioration in performance, causing a decrease in muscular power and endurance and in mental functions [2].

Ginseng is a well-known medicinal herb and has been traditionally used as a medicine for anti-tumor [3], anti-oxidant [4], anti-inflammatory [5] and hypoglycemic properties [6]. Ginseng has also been used to enhance physical strength, expectially in patients who suffered from severe fatigue $[7,8]$ and cancer-related fatigue [9]. Components of ginseng such as ginseng polysaccharide or small molecule oligopeptides isolated from the Panax ginseng have shown anti-fatigue activity and the effects on the physiological biomarkers for fatigue [10] and produced anti-fatigue effect by increasing the forced swimming time and enhancing lactic dehydrogenase (LDH) and glycogen levels in liver of mice [11].

As a type of ginseng, Korean wild ginsengs (SanSam, mountain ginseng) are naturally grown in deep mountains and quite rare and expensive in Korea. To mimic naturally grown ginsengs, ginsengs are often cultivated in deep mountains and classified into SanYangSam (ginseng cultivated in mountain) and SanYangSanSam (wild ginseng cultivated in mountain or cultivated wild ginseng, cWG), depending on the types of ginseng seeds. Crude or distilled extracts from cWG (called as SanYangSanSam) were reported to have anti-tumor or -cancer effect [12, 13]. Especially, the distilled extracts from cWG have been used clinically for injection into acupuncture points in Korea [13] and have also shown to reduce inflammation in lipopolysaccharide (LPS)-induced rat model [14] and oxidative stress in obese rats [15]. While the previous experimental studies have supported the effectiveness of cWG distilled extract, the active components of cWG distilled extract and their biological effects are largely unknown. Recently, by using liquid chromatography tandem mass spectrometry and quadrupole orthogonal acceleration time-of-flight mass spectrometry, we found that cWG distilled extract contained panaxydol as a major component and the level of panaxydol was about 30 times higher than those of ginseng cultivated in mountain (SanYangSam) [16]. As cWG distilled extract has long been used clinically in order to enhance physical energy in cancer patients suffering from fatigue, cWG distilled extract and its major component panaxydol may play a role in reducing fatigue. Thus, the present study was designed to evaluate the anti-fatigue properties of cWG distilled extract and its active component panaxydol in rats by performing the forced swimming test and measuring fatigue-related biomarkers [17] such as blood urea nitrogen (BUN), lactic acid (LAC), $\mathrm{LDH}$, and glycogen in liver and muscle of rats.

\section{Materials and Methods}

\subsection{Animals}

Male Sprague-Dawley rats were purchased from Daehan animal Co. (Seoul, South Korea) weighing 270-320 g and were housed in groups of 2-3 animals per cage at a room temperature of $22 \pm 2^{\circ} \mathrm{C}$ with a $12 \mathrm{hr}$-light-dark cycle and received ad libitum food and water. All experimental procedures were conducted in accordance with National Institutes of Health guidelines for the care and use of laboratory animals and approved by the Institutional Animal Care and Use Committee at Daegu Haany University and Daejeon University.

\subsection{Experimental designs}

Two separated experiments were conducted to test the hypothesis that anti-fatigue properties of cWG distilled extract and its active component panaxydol in rats by performing the forced swimming test and measuring fatigue-related biomarkers such as BUN, LAC, LDH, and glycogen in liver and muscle of rats. The first experiment was conducted to determine whether cWG distilled extract affects forced swimming time and glucogen levels in liver and soleus muscle. Thus, rats were divided into two experimental groups: saline-treated group $(n=6)$ and cWG distilled extract-treated group $(\mathrm{n}=6)$. The second experiment was conducted to see whether panaxydol, an active component of cWG, alters forced swimming time, biochemical levels such as BUN, LAC, and LDH in serum and levels of glycogen in liver and soleus muscel. For this experiment, rats were divided into three experimental groups: vehicle $(\mathrm{n}=5)$, panaxydol $(0.1 \mathrm{mg} / \mathrm{kg})$-treated $(\mathrm{n}=5)$, and panaxydol $(0.25 \mathrm{mg} / \mathrm{kg})$-treated $(\mathrm{n}=5)$ groups.

\subsection{Drugs and chemicals}

Cultivated wild ginseng (cWG, ChonBangNongSan Inc., Chungnam, Korea), about 8-10 years old, was used. The intact cWG was washed, dried, and crushed to super-fine powder (mean particle size, $7.5 \mu \mathrm{m}$ ) using a turbo mill. Distilled extract from cWC (SanYangSanSam, $20 \mathrm{ml} / \mathrm{vial}$ ) was made in Korean Pharmacopuncture Research Institute (KPRI) as described previously [16] 16]. Voucher specimens (\#CWG-2015-03-DE) have been deposited at the KPRI. In brief, the wild ginseng was washed with distilled water to remove debris and contaminants. Fourty grams of the dried wild ginseng were mixed with $250 \mathrm{~mL}$ distilled water in the round plask and heated at $80^{\circ} \mathrm{C}$ via a closed-loop extraction system (KyungSeo machinery Com., Incheon, Korea)(Fig. 1A). During $24 \mathrm{hr}$ heating, the vapor was condensed by cooling in the closed-loop system and cWG distilled extract of about $200 \mathrm{~mL}$ was obtained. Panaxydol was purchased from Chengdu Biopurify Phytochemicals Ltd (Chengdu, China). Levels of BUN, LDH, LAC and glycogen 
were determined with the IDEXX VetTest Chemistry analyzer (IDEXX Laboratories, Westbrook, Maine, USA). Other chemicals were purchased from Sigma Aldrich (St. Louis, MO, USA). Panaxydol was dissolved in $100 \%$ ethanol and then diluted to saline before use. Vehicle, cWG distilled extract $(0.6 \mathrm{~mL} / \mathrm{kg})$ or panaxydol $(0.1$ and $0.25 \mathrm{mg} / \mathrm{kg}$, once a day) was intraperitoneally (i.p.) administered $10 \mathrm{~min}$ prior to the begining of forced swimming test (FST) for 5 days.

\subsection{Forced swimming test}

The force swimming test (FST) was carried out as described previously [1] . Briefly, rats were placed individually into a plastic container $(30 \times 30 \times 80 \mathrm{~cm})$ filled with water $\left(25 \pm 5^{\circ} \mathrm{C}\right)$ to a depth of $60 \mathrm{~cm}$. A glass bar $(10 \%$ of rat's body weight) was attached to the proximal part of the tail of rat. The total swimming time was recorded when the physical strength of rat was exhausted and it could not rise to the surface for more than $10 \mathrm{sec}$.

\subsection{Measurement of blood biochemical parameters}

After last FST on day 5 , blood $(500 \mu \mathrm{L})$ samples were collected from the abdominal aorta under isoflurane anesthesia (2\%) and transferred into heparinized tubes. Serum was obtained by centrifugation at $3000 \mathrm{rpm}$ at $4^{\circ} \mathrm{C}$ for 10 min and stored at $-80^{\circ} \mathrm{C}$ until further analysis. Levels of serum BUN, LAC, and LDH were determined at $562 \mathrm{~nm}$ using a dry slide chemistry Analyzer (VetTest 8008 serum chemistry analyser and VetTest reagent slides, IDEXX Laboratories Inc, Westbrook, Maine, USA) according to the manufacturer's instructions.

\subsection{Measurement of tissue glycogen con- tents}

Rats were euthanized under $4 \sim 5 \%$ isoflurane anesthesia after blood sample collections ( $2 \%$ isoflurane) to obtain liver and soleus mucle tissues. The glycogen levels in liver and soleus mucles were measured by using the method described previously [18] In brief, after sacrificing for blood collection, liver and soleus muscle were quickly dissected out, frozen in liquid nitrogen, and stored at $-80^{\circ} \mathrm{C}$ until use. Each sample (20 mg per tissue) was boiled in 2.0 $\mathrm{M} \mathrm{HCl}$ at $100^{\circ} \mathrm{C}$ for $1 \mathrm{hr}$ and homogenized. After centrifugation, the samples were neutralized with $2.0 \mathrm{M} \mathrm{NaOH}$ and centrifuged again at $3000 \mathrm{rpm}$ for $10 \mathrm{~min}$. Level of glycogen was determined at $562 \mathrm{~nm}$ using a chemistry Analyzer VetTest 8008.

\subsection{Statistical analysis}

Data were carried out using SigmaStat 3 software (Systat Software, Inc, San Jose, CA, USA) and presented as the mean \pm SEM (standard error of mean). Statistical analysis was analyzed by t-test, one-way or two-way repeated analysis of variance (ANOVA), followed by post hoc test using Tukey method. Statistical significance was considered at (*) $P<0.05$ and (**) $P<0.01$.
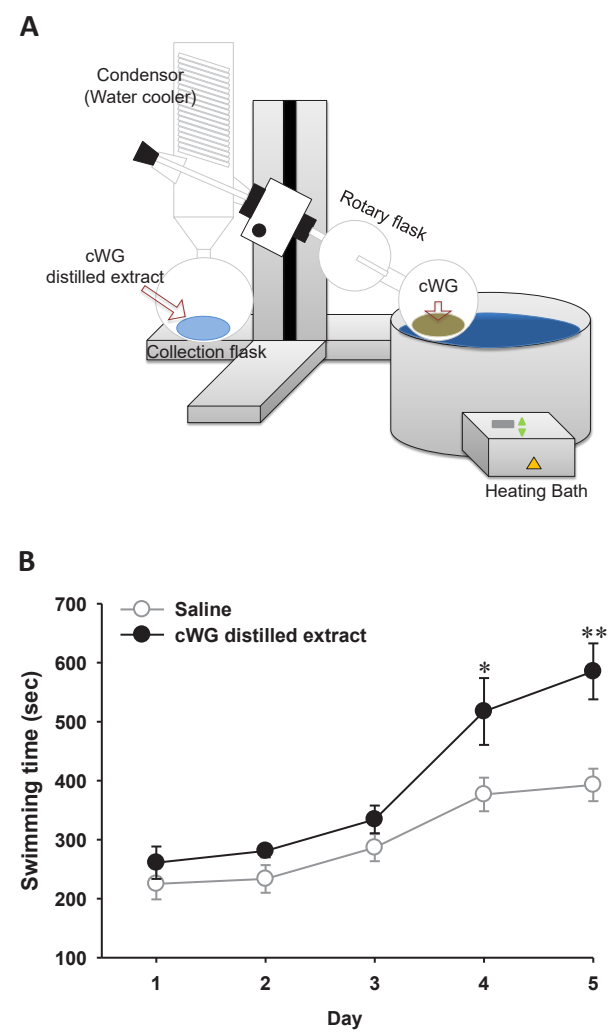

Figure 1 Effect of cultivated wild ginseng (cWG) distilled extract on the forced swimming time in rats.

A: A rotary evaporator used for collection of cWG distilled extract. B: rats were pretreated with saline or cWG distilled extract (0.6 $\mathrm{mL} / \mathrm{kg}$, i.p.) once daily $10 \mathrm{~min}$ prior to the forced swimming test for 5 days. Data were presented as mean $\pm \operatorname{SEM}$ ( $n=6$ per group). ${ }^{*} P<0.05,{ }^{* *} P<0.01$ vs. saline

\section{Results}

\subsection{Effect of cWG distilled extract on the forced swimming test in rats}

The effect of cWG distilled extract on the forced swimming time of rats is shown in Fig.1. The forced swimming time of cWG distilled extract-treated group on day 4 and 5 was significantly longer than that of saline control group (Fig. 1B, repeated t-test; treatment $F_{(1,20)}=10.384, P$ $=0.023$; time $F_{(4,20)}=20.165, P<0.001$; interaction $F_{(4,20)}=$ 2.609, $\mathrm{P}=0.066)$.

\subsection{Effects of cWG distilled extract on gly- cogen in liver or soleus muscle of rats.}

To evaluate whether the effect of cWG distilled extract on forced swimming are associated with glycogen levels, glycogen levels were estimated in liver and soleus muscle of rats after the FST on day 5. As shown in Fig. 2A and 2B, cWG distilled extract did not affect the level of glycogen in liver (Fig. 2A: $t$-test, $F_{(1,10)}=0.056, P=0.82$ ) or soleus muscle 
A

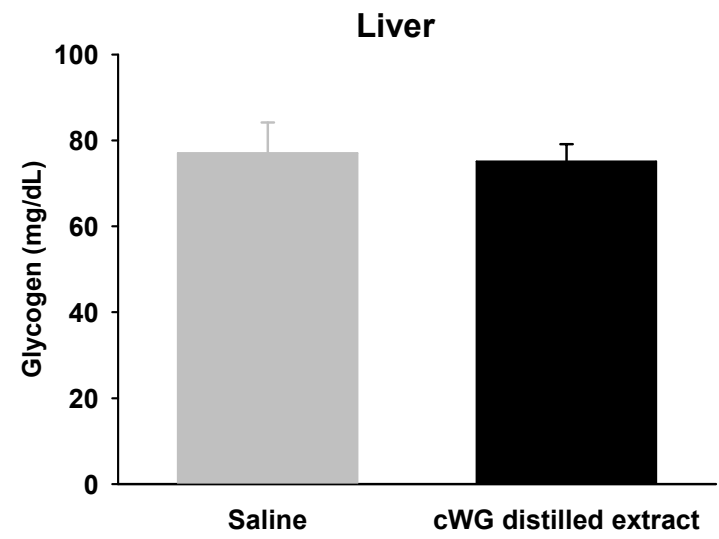

B

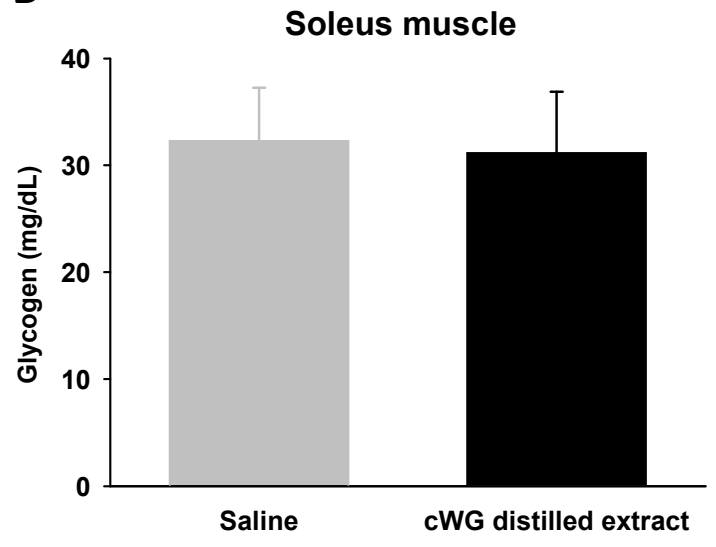

Figure 2 Effect of cultivated wild ginseng (cWG) distilled extract on serum level of glycogen in liver (A) and soleus muscle (B) after forced swimming test.

Rats were treated with saline or cWG distilled extract ( $0.6 \mathrm{~mL} / \mathrm{kg}$, i.p.) for 5 days. A: glycogen content in liver after the forced swimming. B: glycogen content in soleus muscle after the forced swimming. Data were presented as mean \pm SEM ( $n=6$ per group).

A

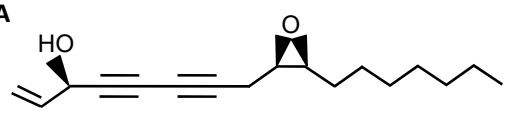

B

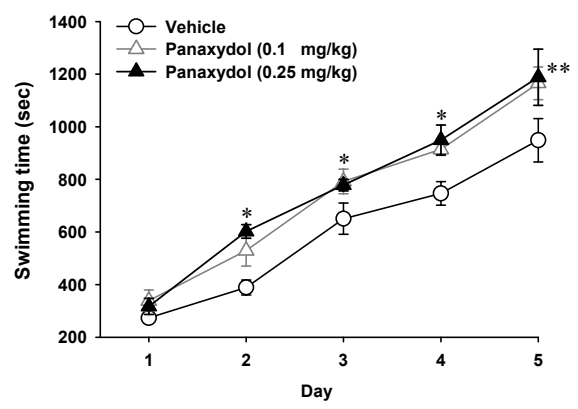

Figure 3 Effect of panaxydol on the forced swimming time in rats. A: chemical structure of panaxydol. B: effect of panaxydol on the forced swimming test. Animals were given vehicle or panaxydol ( 0.1 or $0.25 \mathrm{mg} / \mathrm{kg}$, i.p.) once a day $10 \mathrm{~min}$ prior to the forced swimming test. Data were presented as mean \pm SEM $(n=5$ per group). ${ }^{*} P<0.05,{ }^{* *} P<0.01$ vs. saline.

(Fig. 2B: $t$-test, $\left.F_{(1,10)}=0.023, P=0.88\right)$ compared to saline control group.

\subsection{Effects of panaxydol on the forced swimming test in rats}

We tested the effect of panaxydol, a polyacetylenic compound found in Panax ginseng, on the FST in rats. As shown in Fig. 3B, panaxydol-treated groups $(0.1$ or $0.25 \mathrm{mg} / \mathrm{kg})$ significantly increased forced swimming time compared to vehicle control group on day 4 and 5 (two-way, treatment $\mathrm{F}_{(232)}=21.997, \mathrm{P}<0.001$; time $\mathrm{F}_{(432)}=120.756, \mathrm{P}<$ 0.001 ; interaction $\left.\mathrm{F}_{(8,32)}=1.531, \mathrm{P}=0.186\right)$.

\subsection{Effects of panaxydol on serum bio- chemical parameters of rats}

Levels of fatigue-related serum biomarkers, BUN, LAC, and LDH were measured after last FST. Panaxydol had no effect on level of serum BUN (Fig. 4A; one-way, $\mathrm{F}_{(2,12)}=$ $0.5842, \mathrm{P}=0.0573$ ) or LAC (Fig. 4B; one-way, $\mathrm{F}_{(2,12)}=0.6488$, $\mathrm{P}=0.5401)$. However, LDH level was increased by forced swimming, which was significantly attenuated by treatment with panaxydol at dose of $0.1 \mathrm{mg} / \mathrm{kg}$ for 5 days (Fig. $4 \mathrm{C}$; one-way, $\mathrm{F}_{(2,10)}=10.65, \mathrm{P}=0.003, \mathrm{P}<0.01$ vs. vehicle).
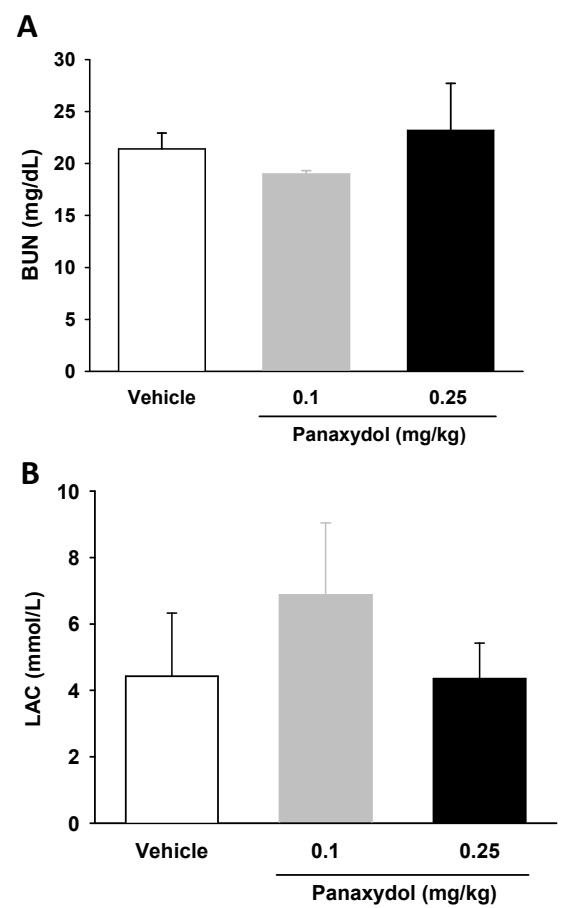
C

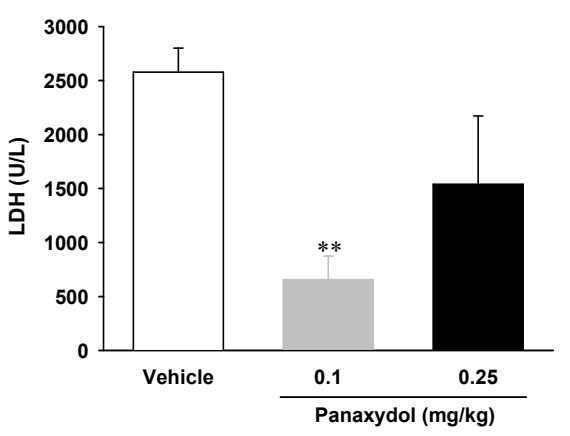

Figure 4 Effect of panaxydol on serum level of fatigue-related biomarkers after forced swimming test.

Rats were pretreated with vehicle or panaxydol ( 0.1 or $0.25 \mathrm{mg}$ / $\mathrm{kg}$, i.p.) 10 min prior to the forced swimming test. A: BUN, B: LAC, $\mathrm{C}$ : LDH after the forced swimming. Data were presented as mean $\pm \operatorname{SEM}\left(n=5\right.$ per group). ${ }^{* *} P<0.01$ vs. vehicle.

\subsection{Effects of panaxydol on glycogen con- tent in liver and soleus muscle tissue after forced swimming}

We also measured the level of glycogen in liver and soleus muscle of rats after the FST on day 5 . As shown in Fig. $5 \mathrm{~A}$ and $5 \mathrm{~B}$, panaxydol had no significant effect on level of glycogen in liver (Fig. 5A: one-way, $\mathrm{F}_{(2,12)}=0.256, \mathrm{P}=0.78$ ) or soleus muscle (Fig. 5B: one-way, $\mathrm{F}_{(2,12)}^{(2,12)}=0.715, \mathrm{P}=0.51$ )
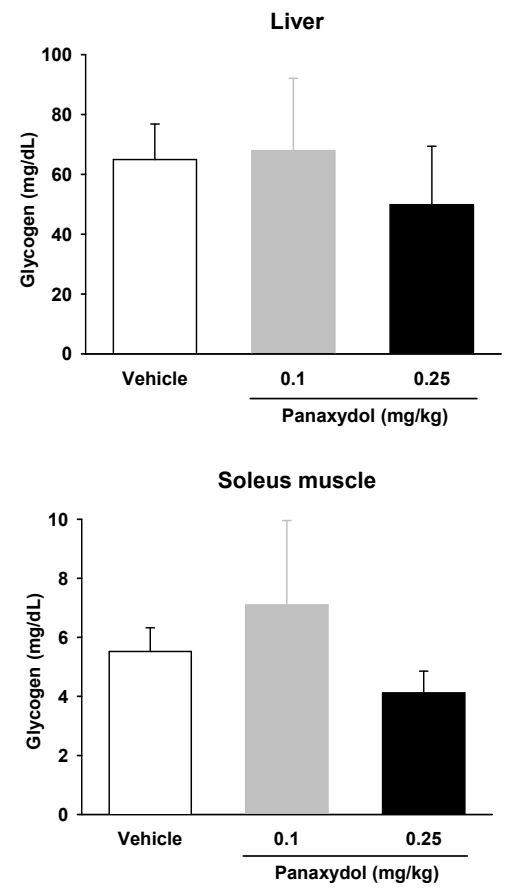

Figure 5 Effect of panaxydol on glycogen content in liver and soleus muscle of rats.

A: glycogen content in liver after the forced swimming. B: glycogen content in soleus muscle after the forced swimming. Data were presented as mean $\pm \operatorname{SEM}$ ( $n=5$ per group). compared to vehicle control group.

\section{Discussion}

Ginseng has various pharmaceutical properties such as anti-tumor [3], anti-oxidant [4] and anti-inflammatory activities [5] and recovery of impaired memory [19]. Panaxidol, a ployacetylene compound isolated from ginseong, has shown a potential anti-cancer agent [20], a protective effct on neurodegeneration in cortical neurons [21], and a mimic the effect of neurve growth factor in PC 12 cells [22] . Several studies have also proved the effectiveness of ginseng or main active components of ginseng, such as ginsenosides $\mathrm{Rbl}$ and small molecule oligopeptieds on exercise fatigue or cancer-related fatigue [11, 23, 24]. Most previous studies have utilized extract ginseng aqueous crude extracts collected through the extraction/condensation steps: ginseng roots are soaked in water or methanol, filtered and condensed by removing excessive water under vaccums. On the other hand, little is known about effects of ginseng distilled (volatile) components produced during evaporizaiton.

The present study shows for the first time, to our knowledge, that distilled extract from ginseng and its active component panaxidol had an anti-fatigue activity in the rat FST model. Fatigue is one of the most common physiological reactions occured from exercise, depression, aging, cancer, multiple sclerosis, and Parkinson's disease. The FST is the most valid models for evaluation of anti-fatigue activity of a wide variety of food or plant compounds [2, 25-27] 2527]. Consistent with a previous study [28] 28], our results revealed that $\mathrm{cWG}$ distilled extract increased the forced swimming time compared to saline control group. In addition, panaxidol, a major component of cWG, also increased the forced swimming time. These result indicates that anti-fatigue effect of ginseng may be associated with the activity of panaxidol in the forced swimming-inducd fatigue.

Exercise-induced fatigue such as forced swimming can be evaluated with biochemical indicatiors, including BUN, LAC and LDH levels in blood. Thus, we measured the level of serum BUN, LAC, and LDH in the rats given forced swimming and panaxydol treatement. Blood urea nitrogen is a metabolic product of proteins and amino acids, used an important indicator for evaluating exercise endurance and fatigue status [29]. Lactate acid is considered a major indicator of muscle fatigue. Intense exercise leads to accummulation of lactate resulting in lowering $\mathrm{pH}$ of blood and muscle and consequent generation of fatigue [30] 30]. Lactate dehydrogenase is an index of muscle damage and catalyzes the interconversion of pyruvate and $\mathrm{NADH}+$ to L-lactate in muscle cells. In our present study, repeated treatments with panaxydol at dose of $0.1 \mathrm{mg} / \mathrm{kg}$ significantly attenuated increased level of serum LDH (major enzyme for lactate production) in forced swimming rats while the levels of serum BUN or LAC were not changed. It may suggest that enhanced performance in forced swimming is associated with LDH. However, it is required to explore how panaxydol can reduce activity of $\mathrm{LDH}$ and muscle damages. Glycogen, which is main storage form of glucose, in liver or muscle is also an index of fatigue [31] 
31]. Glycogen in liver complements the consumption of blood glucose to maintain blood glucose in the physiologic range and fatigue occurs when hepatic glycogen is mostly consumed [32, 33]. In our present study, glycogen levels in liver or soleus muscle of cWG- or panaxydol-treated rats were unaltered. Taken together, these findings suggest that panaxydol may improve physical fatigue via regulation of serum LDH level in forced swimming rats.

As the other possible mechanim, anti-fatigue effect of panaxydol may regulate via attenuation of oxidative stress. It is known that oxidative stress occurs following FST and subsequently may lead to pathology and clinical symptoms of fatigue [34-36]. Bao L et al., has shown that ginseng improved mitochondrial functions and inhibited oxidative stress in skeletal muscles of mice after the FST which may be an action pathway of its anti-fatigue effects [11]. Therefore, panaxydol may have an anti-fatigue effect by reducing the level of oxidative stress indicators such as superoxide dismutase or malondialdehayde in forced swimming model. Further studies should be performed to confirm the mechanisms underlying the anti-fatigue effect of panaxydol on forced swimming-induced fatigue.

\section{Conclusion}

In present study, the forced swimming time in cWG distilled extract-treated group was significantly longer than that of control group on day 4 and 5. Panaxydol-treated groups showed significantly enhanced performance in the forced swimming, compared to control. In addition, a significant decrease in serum LDH level was found in panaxydol-treated group, while there were no alternations in levels of serum BUN and LAC and glycogen in liver or soleus muscle. Taken these results, distilled extract of cultivated wild ginseng and its active component panaxydol produce anti-fatigue activity by reducing activity of $\mathrm{LDH}$ in the rat forced swimming model. some pentacyclic triterpenoids.

\section{Acknowledgment}

The authors have presented the results of this study at the conference (ICMART-iSAMS 2018 World Congress on Medical Acupuncture) and the abstract have cited in Journal of Acupuncture and Meridian Studies (2018; 11(4):239).

\section{Funding}

This study was supported by the Daejeon University fund (201501150001).

\section{Availability of data and materials}

The datasets used and analyzed during the current study available from the corresponding authors (Hee Young Kim, hykim@dhu.ac.kr; Hwa-Seung Yoo, altyhs@dju.kr) on reasonable request.

\section{Authors' contributions}

DHK and ISS performed the experiments and analyzed the data. ISS, DHK, EYJ, HYK, and HSY drafted the manuscript. HYK and HSY were responsible for the overall direction of the project and for edits to the manuscript. All authors have read and agreed on the contents of the manuscript.

\section{Ethics approval and consent to participate}

This study was approved by the institutional Animal Care and Use Committee Daegu Haany University (Daegu, Korea; Approval number: DHU2017-024).

\section{Consent for publication}

Not applicable.

\section{Competing Intersts}

The authors declare that they have no competing interests.

\section{References}

1. Tan W, Yu KQ, Liu YY, Ouyang MZ, Yan MH, Luo R, et al. Anti-fatigue activity of polysaccharides extract from Radix Rehmanniae Preparata. International journal of biological macromolecules. 2012;50(1):59-62.

2. Hao G, Zhang C, Cao W, Hao J. Effects of intragastric administration of five oyster components on endurance exercise performance in mice. Pharmaceutical biology. 2014; 52(6):723-728.

3. Shin HJ, Kim YS, Kwak YS, Song YB, Park JD. Enhancement of antitumor effects of paclitaxel (taxol) in combination with red ginseng acidic polysaccharide (RGAP). Planta medica. 2004;70(11):1033-1038.

4. He LX, Wang JB, Sun B, Zhao J, Li L, Xu T, et al. Suppression of TNF-alpha and free radicals reduces systematic inflammatory and metabolic disorders: Radioprotective effects of ginseng oligopeptides on intestinal barrier function and antioxidant defense. The Journal of nutritional biochemistry. 2017;40:53-61.

5. Yu Q, Zeng KW, Ma XL, Jiang Y, Tu PF, Wang XM. Ginsenoside Rk1 suppresses pro-inflammatory responses in lipopolysaccharide-stimulated RAW264.7 cells by inhibiting the Jak2/Stat3 pathway. Chinese journal of natural medicines. 2017;15(10):751-757.

6. Yun SN, Moon SJ, Ko SK, Im BO, Chung SH. Wild ginseng prevents the onset of high-fat diet induced hyperglycemia and obesity in ICR mice. Archives of pharmacal research. 2004; 27(7):790-796.

7. Banerjee U, Izquierdo JA. Antistress and antifatigue properties of Panax ginseng: comparison with piracetam. Acta Physiol Lat Am. 1982;32(4):277-285.

8. Saito H, Yoshida Y, Takagi K. Effect of Panax Ginseng root on exhaustive exercise in mice. Jpn J Pharmacol. 1974;24(1):119-127. 
9. Park HJ, Shim HS, Kim JY, Park SK, Shim I. Ginseng Purified Dry Extract, BST204, Improved Cancer Chemotherapy-Related Fatigue and Toxicity in Mice. Evidence-based complementary and alternative medicine. 2015;eCAM 2015:197459.

10. Wang J, Li S, Fan Y, Chen Y, Liu D, Cheng H, et al. Anti-fatigue activity of the water-soluble polysaccharides isolated from Panax ginseng C. A. Meyer. Journal of ethnopharmacology. 2010;130(2):421-423.

11. Bao L, Cai X, Wang J, Zhang Y, Sun B, Li Y. Anti-Fatigue Effects of Small Molecule Oligopeptides Isolated from Panax ginseng C. A. Meyer in Mice. Nutrients. 2016;8(12):Article number 807.

12. Min BI, Kim HH, Seo IB, Kwon KR. Antitumor Effects and Protective Effects Against DOxorubicin-induced Testicular Toxicity of Cultivated Wild Ginseng Extract in the B16/F10 Melanoma-Bearing C57BL/6 Mice. Journal of pharmacopuncture. 2007;10(1).

13. Kim K, Choi YS, Joo JC, Moon G. A Case Report for Lung Cancer Patient Showing Remission Treated with Cultivated Wild Ginseng Pharmacopuncture. Journal of Pharmacopuncture. 2011;14(4):33-37.

14. Kim YJ, Lee JM, Lee E. Immunomodulatory activity of cultivated wild ginseng pharmacopuncture. Korean Journal of Acupuncture. 2010;27(1):31-47.

15. Choi EJ, Lee JM, Won SH, Kwon KR. Effects of cultivated wild ginseng pharmacopuncture on lowering lipid and oxidative capacity in biochemical and molecular biological study in obese rats. Journal of Pharmacopuncture. 2006;9(1):5-20.

16. Shin IS, Jo EB, Jang IS, Yoo HS. Quantitative Analyses of the Functional Constituents in SanYangSam and SanYangSanSam. Journal of Pharmacopuncture. 2017;20(4):274-279.

17. Ni W, Gao T, Wang H, Du Y, Li J, Li C, et al. Anti-fatigue activity of polysaccharides from the fruits of four Tibetan plateau indigenous medicinal plants. Journal of ethnopharmacology. 2013;150(2):529-535.

18. Peichuan Z. Analysis of Mouse Liver Glycogen Content. Bio-protocol. 2013;2(10).

19. Dong L, Wang Y, Lv J, Zhang H, Jiang N, Lu C, et al. Memory enhancement of fresh ginseng on deficits induced by chronic restraint stress in mice. Nutritional neuroscience. 2017; 1-8.

20. Guo L, Song L, Wang Z, Zhao W, Mao W, Yin M. Panaxydol inhibits the proliferation and induces the differentiation of human hepatocarcinoma cell line HepG2. Chem Biol Interact. 2009;181(1):138-143.

21. Nie BM, Yang LM, Fu SL, Jiang XY, Lu PH, Lu Y. Protective effect of panaxydol and panaxynol on sodium nitroprusside-induced apoptosis in cortical neurons. Chem Biol Interact. 2006;160(3):225-231.

22. Li WP, Ma K, Jiang XY, Yang R, Lu PH, Nie BM, et al. Molecular mechanism of panaxydol on promoting axonal growth in PC12 cells. Neural regeneration research. 2018;13(11):1927-1936.

23. Tan S, Zhou F, Li N, Dong Q, Zhang X, Ye X et al. Anti-fatigue effect of ginsenoside $\mathrm{Rbl}$ on postoperative fatigue syndrome induced by major small intestinal resection in rat. Biological \& pharmaceutical bulletin. 2013;36(10):1634-1639.
24. Yan B, Liu Y, Shi A, Wang Z, Aa J, Huang X. Investigation of the Antifatigue Effects of Korean Ginseng on Professional Athletes by Gas Chromatography-Time-of-Flight-Mass Spectrometry-Based Metabolomics. Journal of AOAC International. 2018;101(3):701-707.

25. Huang WC, Chiu WC, Chuang HL, Tang DW, Lee ZM, Wei L, et al. Effect of curcumin supplementation on physiological fatigue and physical performance in mice. Nutrients. 2015;7(2):905-921.

26. Tang W, Zhang Y, Gao J, Ding X, Gao S. The anti-fatigue effect of 20(R)-ginsenoside Rg3 in mice by intranasally administration. Biological \& pharmaceutical bulletin. 2008;31(11):2024-2027.

27. Choi EH, Kang JI, Cho JY, Lee SH, Kim TS, Yeo IH, Chun HS. Supplementation of standardized lipid-soluble extract from maca (Lepidium meyenii) increases swimming endurance capacity in rats. Journal of Functional Foods. 2012;4(3):568-573.

28. Ma GD, Chiu CH, Hsu YJ, Hou CW, Chen YM, Huang CC. Changbai Mountain Ginseng (Panax ginseng C.A. Mey) Extract Supplementation Improves Exercise Performance and Energy Utilization and Decreases Fatigue-Associated Parameters in Mice. Molecules. 2017;22(2).

29. Hsiao CY, Hsu YJ, Tung YT, Lee MC, Huang CC, Hsieh CC. Effects of Antrodia camphorata and Panax ginseng supplementation on anti-fatigue properties in mice. The Journal of veterinary medical science. 2018;80(2):284-291.

30. Cairns SP. Lactic acid and exercise performance : culprit or friend? Sports Med. 2006;36(4):279-291.

31. Wang L, Zhang HL, Lu R, Zhou YJ, Ma R, Lv JQ et al. The decapeptide CMS001 enhances swimming endurance in mice. Peptides. 2008;29(7):1176-1182.

32. Rahman M, Yang DK, Kim GB, Lee SJ, Kim SJ. Nigella sativa seed extract attenuates the fatigue induced by exhaustive swimming in rats. Biomedical reports. 2017;6(4):468-474.

33. Jia JM, Wu CF. Antifatigue Activity of Tissue Culture Extracts of Saussurea involucrata. Journal of Pharmoceutical Biology. 2008;46(6):433-436.

34. Wu C, Chen R, Wang XS, Shen B, Yue W, Wu Q. Antioxidant and anti-fatigue activities of phenolic extract from the seed coat of Euryale ferox Salisb. and identification of three phenolic compounds by LC-ESI-MS/MS. Molecules. 2013;18(9):11003-11021.

35. Wang J, Sun C, Zheng Y, Pan H, Zhou Y, Fan Y. The effective mechanism of the polysaccharides from Panax ginseng on chronic fatigue syndrome. Archives of pharmacal research. 2014;37(4):530-538.

36. Barclay JK, Hansel M. Free radicals may contribute to oxidative skeletal muscle fatigue. Canadian journal of physiology and pharmacology. 1991;69(2):279-284. 